\title{
Irrupção do primeiro molar permanente e o peso da criança. Há uma associação?
}

\author{
Eruption of the first permanent molar and the child's weight. Is there an association? \\ Erupción del primer molar permanente y peso del niño. ¿ Existe alguna associación?
}

Recebido: 16/02/2021 | Revisado: 21/02/2021 | Aceito: 17/03/2021 | Publicado: 22/03/2021

Eduarda Verônica Tavares do Couto
ORCID: https://orcid.org/0000-0002-2284-8526
Universidade Metropolitana de Santos, Brasil
E-mail: dudaengmec@gmail.com
Marjory Inácio Franco
ORCID: https://orcid.org/0000-0002-7369-2971
Universidade Metropolitana de Santos, Brasil
E-mail: marjory_i.f@ hotmail.com
Sandra Kalil Bussadori
ORCID: https://orcid.org/0000-0002-9853-1138
Universidade Metropolitana de Santos, Brasil
E-mail: sandra.skb@gmail.com
Elaine Marcílio Santos
ORCID: https://orcid.org/0000-0003-1084-9940
Universidade Metropolitana de Santos, Brasil
E-mail: elaine.marcilio@unimes.br
Karla Mayra Rezende
ORCID: https://orcid.org/0000-0003-4340-0699
Universidade Metropolitana de Santos, Brasil
E-mail: karla-rezende@hotmail.com

\section{Resumo}

A erupção dentária é um dos fenômenos que se manifesta como parte do crescimento e desenvolvimento da criança. Vários fatores podem estar relacionados com o momento de irrupção dos dentes permanentes decorrentes de múltiplas causas que variam de ordem genéticas, ambientais, étnicos e raciais. O objetivo desta pesquisa foi verificar se há uma associação do peso da criança com erupção do primeiro molar permanente. A pesquisa analisou 224 crianças residente na baixada santista do Estado de São Paulo com idade entre 5 a 7 anos. O exame clinico foi realizado na cadeira odontógica, foi considerado presença de molar irrompido qualquer parte da coroa que rompeu a gengiva e era visível na cavidade bucal e em qualquer quadrante. Após o exame as crianças eram pesadas descalças e sem a camiseta. Os resultados mostraram que o peso não apresentou uma influência na erupção, e que as crianças do gênero feminino tiveram erupção mais precoce quanto comparado com os meninos.

Palavras-chave: Erupção dentária; Estado nutricional; Dente molar.

\begin{abstract}
Tooth eruption is one of the manifest as part of the child's growth and development. Several factors can be related to the moment of eruption of permanent teeth resulting from multiple causes that vary from genetic, environmental, ethnic and racial orders. The objective of this research was to verify if there is an association of the child's weight with eruption of the first permanent molar. The research analyzed 224 children residing in the Santos region of the State of São Paulo aged between 5 and 7 years. The clinical examination was performed in the dental chair, the presence of an erupted molar was considered to be any part of the crown that ruptured the gingiva and was visible in the oral cavity and in any quadrant. After the exam, the children were heavy barefoot and without a T-shirt. This results showed that the weight did not include an influence on the tooth eruption, and that in the group studied, female children had earlier eruption than boys.
\end{abstract}

Keywords: Tooth eruption; Nutritional status; Molar tooth.

\section{Resumen}

La erupción dental es uno de los fenómenos que se manifiesta como parte del crecimiento y desarrollo del niño. Varios factores pueden estar relacionados con el momento de la erupción de los dientes permanentes como resultado de múltiples causas que varían desde genéticas, ambientales, étnicas y raciales. El objetivo de esta investigación fue verificar si existe una asociación del peso del niño con la erupción del primer molar permanente. La investigación analizó a 224 niños residentes en la región de Santos del estado de São Paulo con edades entre 5 y 7 años. El examen clínico se realizó en el sillón dental, se consideró la presencia de un molar erupcionado a cualquier parte de la corona que rompiera la encía y fuera visible en la cavidad bucal y en cualquier cuadrante. Después del examen, los niños 
estaban descalzos y sin camisa. Los resultados mostraron que el peso no influyó en la erupción y que las niñas tenían una erupción antes que los niños.

Palabras clave: Erupción dentaria; Estados nutricionales; Diente molar.

\section{Introdução}

Os odontopediatras frequentemente se deparam com situações clinicas que envolvem anomalias ou atraso na erupção dentária (Sanchez \& Ubios, 2020). A literatura considera processo de erupção dentária como um indicador de crescimento e desenvolvimento devido a regularidade com que os dentes irrompem na cavidade bucal (K. M. Rezende, Zöllner, \& Santos, 2010). A primeira utilização da erupção dentária como indicador de medida de crescimento ocorreu no século XIX, na Inglaterra, quando um relatório industrial estipulou que crianças que não apresentassem o segundo molar permanente não poderiam trabalhar nas fábricas (Barberia Leache, Maranes Pallardo, Mourelle Martinez, \& Moreno Gonzalez, 1988; Duque et al., 2004).

Desde então, a erupção dentária tem sido considerado um dado complementar útil para avaliação de crescimento da criança, principalmente quando empregado em conjunto com outras medidas como o peso corporal e ou em conjunto com o desenvolvimento esquelético a partir da radiografia carpal (De Angelis et al., 2014; Haddad \& Correa, 2005; PÊGO et al., 2021).

O primeiro molar permanente irrompem na cavidade bucal a partir dos 5 anos de idade e leva em média, 15 meses para entrar em oclusão com seu antagonista, estando durante esse período sob o risco aumentado de desenvolver lesões de cárie devido à forte relação entre o grau de erupção e o acumulo de biofilme na superfície (Ekstrand, Christiansen, \& Christiansen, 2003). Os primeiros molares permanentes são conhecidos como a chave para formação do arco permanente e detêm os maiores esforços oclusais. A importância deste dente reside na sua função adequada da mastigação, oclusão e harmonia dento facial (Saber et al., 2018).

Fatores como etnia, sexo, alterações sistêmicas, aspectos socioeconômicos, clima, estado nutricional, obesidade e prematuridade podem influenciar no tempo da irrupção dentária de dentes permanentes (De Souza, Manju, \& Hegde, 2018; Evangelista et al., 2018; Garmash, 2019; Holloway, 1965).

O presente estudo teve como objetivo correlacionar o peso corporal de crianças com idade entre 5 a 7 anos com a irrupção do primeiro molar permanente.

\section{Metodologia}

A presente pesquisa foi aprovada pelo Comitê de Ética e Pesquisa e está registrada sob número CAAE: 17238919.0.0000.5509

Trata-se de um estudo quantitativo, descritivo e transversal.

O período da coleta dos dados foi nos meses de dezembro 2019, janeiro e fevereiro de 2020 . O total da amostra foi de 234 crianças sendo 120 do sexo masculino $(51,28 \%)$ e 114 do sexo feminino (48,72\%), com idade entre 5 a 7 anos atendidas na clinica infantil da Universidade Metropolitana de Santos (UNIMES). Após o consentimento dos pais, as crianças eram incluídas na pesquisa. Foram excluídas as crianças que não estavam acompanhadas dos pais ou responsáveis, pois o termo de consentimento livre e esclarecido necessitava ser assinado, autorizando a inclusão da criança na pesquisa; aquelas que não estavam na faixa etária preconizada e que apresentavam alguma doença sistêmica.

A idade foi calculada a partir de a data de nascimento de cada criança até à data do exame e arredondado para o mês inteiro. 
Duas alunas de graduação em Odontologia realizaram o exame clínico bucal com auxilio de espátula de madeira com objetivo de verificar a presença ou ausência de algum primeiro molar (superior ou inferior, direito e esquerdo). Os dentes foram identificados de acordo com o sistema de dois dígitos do Federação Dentaire Internationale (FDI). O estado de erupção de cada dente permanente era registado. Mesmo que uma pequena parte da coroa seja visível clinicamente penetrando a membrana mucosa oral, foi considerado em erupção e registado em conformidade. Avaliação radiográfica não foi realizada.

No box do exame clinico havia uma balança digital portátil tech line, no qual cada participante, era pesado descalço. $\mathrm{O}$ peso e a presença do primeiro molar permanente foram tabulados no programa excel.

\section{Resultados}

Com o intuito de analisar os resultados, os dados obtidos foram agrupados em tabelas e gráficos. Informações como data de nascimento, sexo, presença ou ausência do primeiro molar permanente foram compilados em planilhas do software Microsoft® Excel 2019 para análise comparativa.

\section{Teste de Confiança}

Não houve tentativa de avaliar o erro do método de registro da erupção dentária porque os critérios da irrupção dentária são bem definidos: presente ou ausente.

Gráfico 1: Porcentagem dos primeiros molares que já haviam irrompidos.

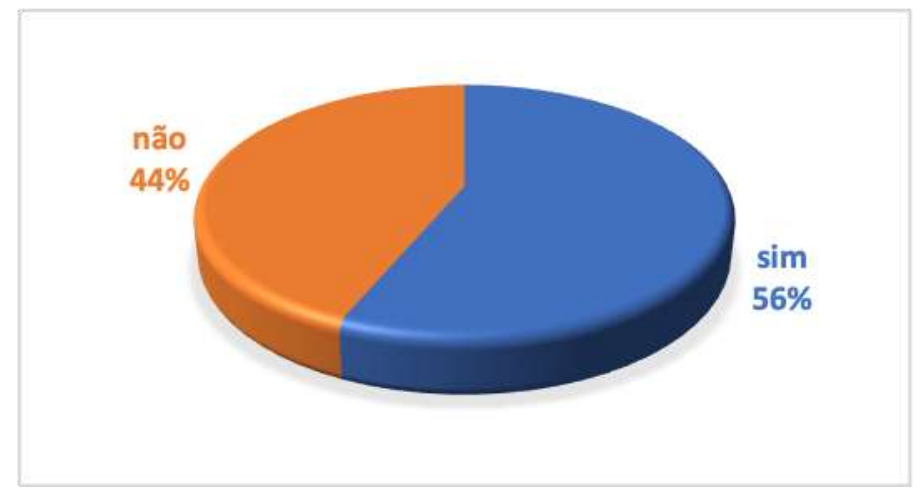

Fonte: Autores.

Do total de crianças que participaram da pesquisa, independente do sexo, peso e idade, $56 \%$ apresentavam o primeiro molar irrompido na cavidade bucal. 
Gráfico 2: Número de crianças de acordo com idade e sexo.

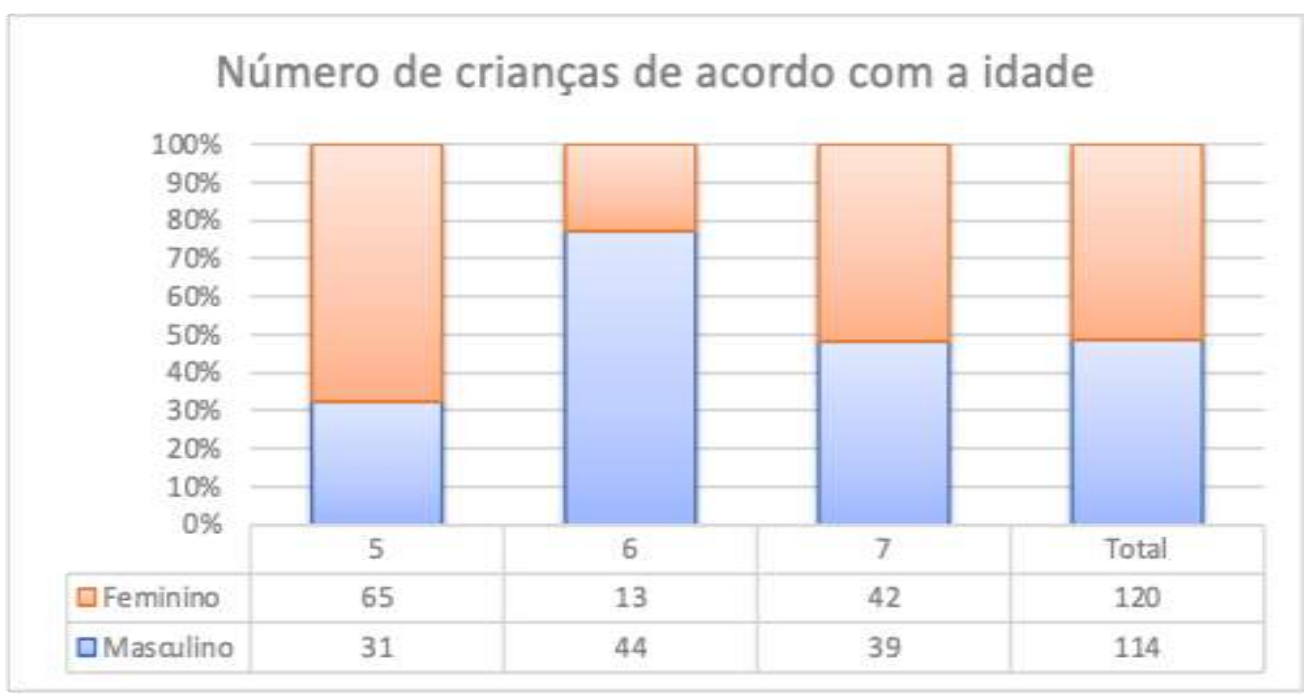

Fonte: Autores.

De acordo com a Organização Mundial de saúde, criança na faixa etária escolhida do trabalho, apresenta a seguinte variação de peso x idade x sexo (Organização Mundial da Saúde).

Tabela 1: Relação entre idade x peso de acordo com a Organização Mundial da Saúde (O.M.S)

\begin{tabular}{|l|l|l|l|l|}
\hline Idade & \multicolumn{1}{|c|}{ Masculino (kg) } & \multicolumn{1}{|c|}{ Média meninos } & \multicolumn{1}{|c|}{ Feminino (kg) } & \multicolumn{1}{|c|}{ Média meninas } \\
\hline 5 anos & $16,26-21,07$ & $18,66 \mathrm{~kg}$ & $15,41-21,71$ & $18,56 \mathrm{~kg}$ \\
\hline 6 anos & $17,93-24,14$ & $21,03 \mathrm{~kg}$ & $17,06-24,28$ & $29,67 \mathrm{~kg}$ \\
\hline 7 anos & $19,72-27,44$ & $23,58 \mathrm{~kg}$ & $18,85-26,94$ & $17,89 \mathrm{~kg}$ \\
\cline { 2 - 5 } & &
\end{tabular}

Fonte: Autores.

A Tabela 2 apresenta correlação entre o peso, idade e presença do primeiro molar permanente das crianças participantes da pesquisa. A média de peso no grupo dos meninos com idade de 05 anos foi de $20,13 \mathrm{~kg}$ com desvio padrão de 4.30. Nesse grupo 12 crianças apresentaram primeiro molar permanente. Com as crianças com 6 anos, 18 apresentaram erupção contra 26 crianças que não apresentaram irrupção e com média de peso semelhante com a recomendada pela O.M.S. Quando observamos nas crianças do sexo masculino com 7 anos, a média de peso estava abaixo daquela recomendada pela O.M.S, porém 24 crianças haviam a presença do primeiro molar contra 15 crianças. 
Tabela 2: Relação entre idade x peso de acordo com a Organização Mundial da Saúde (O.M.S) e média do grupo estudos de crianças do sexo masculino.

\begin{tabular}{|c|c|c|c|c|c|}
\hline Idade & $\begin{array}{c}\text { Meninos média } \\
\text { peso O.M.S. }\end{array}$ & $\begin{array}{c}\text { Média peso } \\
\text { grupo }\end{array}$ & Desvio padrão & $\begin{array}{c}\text { Erupcionado 1 } \\
\text { molar }\end{array}$ & $\begin{array}{c}\text { Não erupcionado } \\
\text { o 1 molar }\end{array}$ \\
\hline 5 anos & $18,66 \mathrm{~kg}$ & $20,13 \mathrm{~kg}$ & 4,30 & 12 & 19 \\
\hline 6 anos & $21,03 \mathrm{~kg}$ & $21,11 \mathrm{~kg}$ & 4,02 & 26 & 18 \\
\hline 7 anos & $23,58 \mathrm{~kg}$ & $22,25 \mathrm{~kg}$ & 5,31 & 24 & 15 \\
\hline
\end{tabular}

Fonte: Autores.

Tabela 3: Relação entre idade x peso de acordo com a Organização Mundial da Saúde (O.M.S) e média do grupo estudos de crianças do sexo feminino.

\begin{tabular}{|c|c|c|c|c|c|}
\hline Idade & $\begin{array}{c}\text { Meninas média } \\
\text { peso O.M.S. }\end{array}$ & $\begin{array}{c}\text { Média peso } \\
\text { grupo }\end{array}$ & $\begin{array}{c}\text { Desvio } \\
\text { padrão }\end{array}$ & $\begin{array}{c}\text { Erupcionado } \\
\mathbf{1} \text { molar }\end{array}$ & $\begin{array}{c}\text { Não erupcionado } \\
\text { 0 1 molar }\end{array}$ \\
\hline 5 anos & $18,56 \mathrm{~kg}$ & $20,64 \mathrm{~kg}$ & 3,81 & 41 & 24 \\
\hline 6 anos & $29,67 \mathrm{~kg}$ & $20,48 \mathrm{~kg}$ & 2,69 & 07 & 06 \\
\hline 7 anos & $17,89 \mathrm{~kg}$ & $21,6 \mathrm{~kg}$ & 5,65 & 22 & 20 \\
\hline
\end{tabular}

Fonte: Autores.

No grupo das meninas, as meninas com 5 anos apresentaram peso acima da média recomendada pela organização mundial da saúde e 41 crianças apresentaram irrupção contra 24. No grupo de 6 anos, a média de peso do grupo de pesquisa foi abaixo à média estipulada. Nesse grupo 07 meninas apresentaram erupção contra 6. Por fim, o grupo dos 7 anos de meninas a média do peso foi acima da recomendação da O.M.S. e neste grupo 22 haviam primeiro molar permanente contra 20.

\section{Discussão}

Os primeiros molares permanentes fazem parte de um grupo de dentes denominados monofísários, porque não sucedem a um dente deciduo e nem são substituídos por qualquer outro dente e irrompem na cavidade bucal de forma silenciosa e assintomática e por terem uma anatomia peculiar podem acumular biofilme favorecendo a progressão da doença cárie. Além de serem importante quando pensamos na chave de oclusão quando há perda precoce pode alterar a curva de spee, a ATM (Bakhurji, Gaffar, Nazir, Al-Khalifa, \& Al-Ansari, 2020; Ekstrand et al., 2003; Saber et al., 2018). O primeiro molar permanente também é conhecido como molar dos 6 anos. A irrupção precoce ou tardia é definida com base nesta idade de referencia (Hovorakova, Lesot, Peterka, \& Peterkova, 2018; Jenkins, 2017; Karla Mayra Rezende, Côrrea, Côrrea, \& Bönecker, 2013). Mesmo sendo um assunto frequentemente estudado, não encontramos nenhum dado em relação as crianças que vivem na cidade de Santos-SP, o que motivou a iniciar essa pesquisa.

Na medicina pediátrica, o hábito de observar a dentição, juntamente com peso e a altura, podem ajudar a diagnosticar ou apontar problemas de desenvolvimento da criança além de doenças e ou sugerir problemas nutricionais e consequências de prematuridade (Gibson \& Conchie, 1964; Karla Mayra Rezende et al., 2013; K. M. Rezende et al., 2010).

Neste trabalho, a metodologia para avaliar as crianças foi o método transversal. Esse é método preferível ao método longitudinal, pois é possível incluir amostras maiores, produzindo resultados mais representativos da população. Além disso, o risco de viés é menor do que nos estudos longitudinais (Lakshmappa, Guledgud, \& Patil, 2011; K. M. Rezende et al., 2010). 
Nas crianças analisadas, as meninas (57\%) apresentaram o irrompimento do primeiro molar mais precoce quanto observamos no grupo dos meninos $(26 \%)$.

Em relação ao peso corpóreo nas crianças analisadas, todas estavam na média de peso considerada adequada pela O.M.S. Porém no grupo das meninas com idade de 5 anos estavam com média de peso de 2,08 $\mathrm{kg}$ a mais da recomendada pela O.M.S. bem como aquelas com idade de 7 anos, a média de peso estava 3,71 kg a mais. Já no grupo de 6 anos, a média de peso estava abaixo de 9,19 kg, porém mesmo assim 07 crianças já apresentaram erupção dentária contra 06 crianças pertencente ao mesmo grupo etário. No grupo dos meninos, mesmo as crianças com 5 anos estavam 1,47 $\mathrm{kg}$ acima do recomendado, 19 crianças não apresentavam o primeiro molar permanente. Já no grupo de 7 anos, mesmo a média de peso estarem abaixo 1,33 $\mathrm{kg}$ do recomendado pela O.M.S, 24 crianças apresentavam o molar. Ou seja, no nosso grupo de crianças, o peso não foi fator constatar irrupção precoce ou atraso. Isso pode ser explicado porque a variação do peso corpóreo com a idade no grupo de pesquisa não foi considerado crianças desnutridas ou com obesidade. No entanto, a literatura evidencia uma correlação positiva entre sobrepeso/obesidade e erupção precoce nos dentes permanentes (Zaidi, Thayath, Singh, \& Sinha, 2015) e do contrário, quando crianças desnutridas tendem a ter atraso na erupção (Heinrich-Weltzien, Zorn, Monse, \& Kromeyer-Hauschild, 2013). Portanto, usar o peso corporal para comparar e erupção não foi possível. Assim, baseado nestes resultados, recomenda-se continuar o trabalho agora estipulando grupos iguais para cada faixa etária, sexos e até mesmo crianças com peso considerado normal, desnutrido e com obesidade pela organização mundial da saúde para novas associações para confirmar os resultados aqui apresentados.

Em resumo, a chave para o manejo clinico consiste em compreender os fatores que influenciam na irrupção dentária para o acompanhamento e desenvolvimento da criança é fundamental a todo profissional de saúde. É importante que o profissional se atualizem sempre e tenham em mente que as variáveis ambientais como alimentação, hábitos culturais, prática de aleitamento materno e regionalismos podem influenciar no tempo da irrupção dentária e consequentemente no padrão mastigatório da criança.

\section{Conclusão}

A faixa etária da erupção do primeiro molar permanente foi de 6 anos no grupo dos meninos e 5 anos no grupo das meninas.

A média do peso das crianças estavam de acordo com idade com os dados recomendado pela O.M.S. não conseguindo, portanto, associar o peso com aceleração ou atraso da irrupção do primeiro molar.

Novos estudos colocando grupo de crianças com obesidades, normais e desnutridas são necessários.

\section{Referências}

Bakhurji, E., Gaffar, B., Nazir, M., Al-Khalifa, K., \& Al-Ansari, A. (2020). First Permanent Molar Caries and Oral Health Practices in Saudi Male Teenagers: Inequalities by Socioeconomic Position. Scientifica (Cairo), 2640949. 10.1155/2020/2640949

Barberia Leache, E., Maranes Pallardo, J. P., Mourelle Martinez, M. R., \& Moreno Gonzalez, J. P. (1988). Tooth eruption in children with growth deficit. J Int Assoc Dent Child, 19(2), 29-35. https://www.ncbi.nlm.nih.gov/pubmed/3273301

De Angelis, D., Gibelli, D., Merelli, V., Botto, M., Ventura, F., \& Cattaneo, C. (2014). Application of age estimation methods based on teeth eruption: how easy is Olze method to use? Int J Legal Med, 128(5), 841-844. 10.1007/s00414-014-1006-0

De Souza, N., Manju, R., \& Hegde, A. M. (2018). Development and evaluation of new clinical methods of age estimation in children based on the eruption status of primary teeth. J Indian Soc Pedod Prev Dent, 36(2), 185-190. 10.4103/JISPPD.JISPPD_121_17

Duque, C., Dalben Gda, S., Aranha, A. M., Carrara, C. F., Gomide, M. R., \& Costa, B. (2004). Chronology of deciduous teeth eruption in children with cleft lip and palate. Cleft Palate Craniofac J, 41(3), 285-289. 10.1597/02-091.1

Ekstrand, K. R., Christiansen, J., \& Christiansen, M. E. (2003). Time and duration of eruption of first and second permanent molars: a longitudinal investigation. Community Dent Oral Epidemiol, 31(5), 344-350. 10.1034/j.1600-0528.2003.00016.x 
Research, Society and Development, v. 10, n. 3, e47410313024, 2021

(CC BY 4.0) | ISSN 2525-3409 | DOI: http://dx.doi.org/10.33448/rsd-v10i3.13024

Evangelista, S. E. S., Vasconcelos, K. R. F., Xavier, T. A., Oliveira, S., Dutra, A. L. T., Nelson-Filho, P., \& Kuchler, E. C. (2018). Timing of Permanent Tooth Emergence is Associated with Overweight/Obesity in Children from the Amazon Region. Braz Dent J, 29(5), 465-468. 10.1590/0103-6440201802230

Garmash, O. (2019). Dependence of Deciduous Tooth Eruption Terms and Tooth Growth Rate on the Weight-Height Index at Birth in Macrosomic Children over the First Year of Life. Acta Medica (Hradec Kralove), 62(2), 62-68. 10.14712/18059694.2019.48

Gibson, W. M., \& Conchie, J. M. (1964). Observation of Children's Teeth as a Diagnostic Aid: A Review. I. Dentition in the Assessment of Development. Can Med Assoc J, 90, 70-75. https://www.ncbi.nlm.nih.gov/pubmed/14104154

Haddad, A. E., \& Correa, M. S. (2005). The relationship between the number of erupted primary teeth and the child's height and weight: a cross-sectional study. J Clin Pediatr Dent, 29(4), 357-362. 10.17796/jcpd.29.4.j10510371q155847

Heinrich-Weltzien, R., Zorn, C., Monse, B., \& Kromeyer-Hauschild, K. (2013). Relationship between malnutrition and the number of permanent teeth in Filipino 10- to 13-year-olds. Biomed Res Int, 2013, 205950. 10.1155/2013/205950

Holloway, P. J. (1965). The Effect of Nutrition on Tooth Development. $R$ Soc Health $J, \quad 85, \quad 113-116 \quad$ PASSIM. https://www.ncbi.nlm.nih.gov/pubmed/14295171

Hovorakova, M., Lesot, H., Peterka, M., \& Peterkova, R. (2018). Early development of the human dentition revisited. J Anat, 233(2), 135-145. 10.1111/joa. 12825

Jenkins, D. (2017). Development of the human dentition. Cranio, 35(1), 64. 10.1080/08869634.2016.1258982

Lakshmappa, A., Guledgud, M. V., \& Patil, K. (2011). Eruption times and patterns of permanent teeth in school children of India. Indian J Dent Res, 22(6), 755-763. $10.4103 / 0970-9290.94568$

Pêgo, M. d. M. F., Corazza, P. F. L., Baeder, F. M., Furtado SILVA, D., Albuquerque, A. C. L. d., Junqueira, J. L. C., \& Panzarella, F. K. (2021). Development of the teeth, cervical vertebrae, hand and wrist combined for the estimation of the biological age. Research, Society and Development, 10(3), 12. $10.33448 /$ rsd-v10i3.12948.

Rezende, K. M., Côrrea, M. S. N. P., Côrrea, F. N. P., \& Bönecker, M. (2013). Erupção dentária decidua. Cronologia, manifestações locais e sistêmicas. In Napoleão (Ed.), Anuário de Odontopediatria- Integrada e Atual (Vol. 1). Campinas: 2013.

Rezende, K. M., Zöllner, M. S. A. C., \& Santos, M. R. N. (2010). Avaliação da erupção dentária decídua em bebês considerados de risco. Pesquisa Brasileira Odontoped Clin Integr, 10(1), 5.

Saber, A. M., Altoukhi, D. H., Horaib, M. F., El-Housseiny, A. A., Alamoudi, N. M., \& Sabbagh, H. J. (2018). Consequences of early extraction of compromised first permanent molar: a systematic review. BMC Oral Health, 18(1), 59. 10.1186/s12903-018-0516-4

Sanchez, L. M., \& Ubios, A. M. (2020). Alterations in odontogenesis and tooth eruption resulting from exposure to hexavalent chromium in suckling animals. Int J Paediatr Dent, 30(1), 35-41. 10.1111/ipd.12573

Zaidi, I., Thayath, M. N., Singh, S., \& Sinha, A. (2015). Preterm Birth: A Primary Etiological Factor for Delayed Oral Growth and Development. Int J Clin Pediatr Dent, 8(3), 215-219. 10.5005/jp-journals-10005-1316 